\title{
Traducción al inglés de «Abbott y Costello", de Sergio Ramírez: una perspectiva terminológica ${ }^{1}$
}

\section{(English Translation of Sergio Ramírez's "Abbott y Costello": A Terminological Approach)}

\section{Lindsay Chaves Fernández²}

Universidad Nacional, Heredia, Costa Rica

\section{Henry Sevilla Morales ${ }^{3}$}

Universidad Nacional, Heredia, Costa Rica

Universidad de Costa Rica, San Ramón, Costa Rica

\begin{abstract}
Resumen
Consiste en una propuesta de traducción al inglés del cuento «Abbott y Costello», del nicaragüense Sergio Ramírez. Es un trabajo terminológico que comprende la selección, búsqueda en textos paralelos y traducción al inglés de cincuenta términos problemáticos, según lo recomendado en el Manual de gestión terminológica, de Sherry E. Gapper. Se presenta además un análisis traductológico de la terminología seleccionada y se esbozan algunas conclusiones. Finalmente, se analizan implicaciones teóricas y prácticas y se dan recomendaciones para la traducción de textos literarios.
\end{abstract}

1 Recibido: 10 de abril de 2020; aceptado: 5 de abril de 2021.

2 Escuela de Literatura y Ciencias del Lenguaje; https://orcid.org/0000-0001-7936-8112. Correo electrónico: lindsay.chaves.fernandez@una.ac.cr

3 Escuela de Literatura y Ciencias del Lenguaje; https://orcid.org/0000-0003-4040-8062. Correo electrónico: henry.sevilla.morales@una.ac.cr 


\begin{abstract}
The current paper proposes an English translation of the short story "Abbott y Costello", by the Nicaraguan Sergio Ramírez. To this end, a terminological approach was used in the selection, search in equivalent texts, and English translation of 50 complex terms, based on the recommendations in Sherry E. Gapper's Manual de gestión terminológica. In addition, a translationbased analysis is presented for the selected terms, along with concluding remarks. Implications for theory and practice are also discussed and recommendations for literary translation are provided.
\end{abstract}

Palabras clave: traducción literaria, terminología, crónica, estilo periodístico, registro literario, literatura nicaragüense

Keywords: literary translation, terminology, chronicle, journalistic style, literary register, Nicaraguan literature

[...] si tú no emigraste, emigró tu padre, y si tu padre no necesitó mudar de sitio fue porque tu abuelo, antes que él, no tuvo otro remedio que irse, cargando la vida sobre las espaldas, en busca del pan que su tierra le negaba.

José Saramago ${ }^{4}$

\title{
Introducción
}

En 2005, la muerte del nicaragüense Natividad Canda Mairena por el ataque de unos feroces perros rottweilers estremeció a la sociedad y desató la crítica de la ciudadanía, debido tanto a lo sangriento del suceso como a la impasibilidad de los testigos que lo contemplaron. Según fuentes oficiales, el hecho ocurrió pasada la medianoche del 10 de noviembre, cuando Canda Mairena, de 25 años, se disponía a robar en un taller automotriz en Cartago. Tanto el dueño del taller como los guardas de seguridad y los agentes de la Fuerza Pública se habrían mostrado indiferentes a los hechos que terminaron con 197 mordiscos en el cuerpo de la víctima y su muerte por shock hipovolémico. Luego de un complejo proceso judicial, los imputados resultaron absueltos,

4 José Saramago, El último cuaderno: textos escritos para el blog. Marzo de 2009 - junio de 2010 (Alfaguara, 2011) [versión consultada: libro de Kindle]. 
lo que contribuyó a intensificar el rechazo y la xenofobia entre la población de ambos países 5 .

A raíz de lo acontecido, Sergio Ramírez escribe el cuento «Abbott y Costello», tomando como base los recursos de la crónica periodística, en el que de manera factual expone los hechos que llevaron a la muerte de Canda Mairena. Para revestir la obra de veracidad, presenta los acontecimientos, prescindiendo de juicios de valor o críticas directas a los personajes o al contexto donde se enmarcan los hechos. No obstante, como nada en la literatura puede darse con objetividad, de forma sutil, el autor secuencia los hechos para urdir una fulminante crítica al accionar de quienes pudieron haber impedido una muerte brutal.

En este marco referencial, el presente trabajo propone una traducción al inglés del cuento «Abbott y Costello», utilizando un enfoque terminológico, así como estrategias de traducción acordes con los elementos de la crónica periodística, subgénero narrativo utilizado por Ramírez en el texto original (TO). Se empleó la siguiente metodología: (1) se realizó un análisis textual y extratextual para delimitar el TO socioculturalmente; (2) se estudiaron textos paralelos en la lengua meta a fin de procurar un estilo equivalente al del TO y, la vez, encontrar las equivalencias terminológicas necesarias para la traducción; (3) se seleccionaron 50 términos problemáticos de traducir y se crearon las fichas conceptuales, terminológicas y bibliográficas, según lo recomendado en el Manual de gestión terminológica ${ }^{6}$ y (4) se comparó el texto traducido (TT) con el TO para verificar la fidelidad de la traducción según el enfoque terminológico seleccionado.

5 Ver: César Villegas, «"Entre la risa y el odio”. Una aproximación a la xenofobia en la producción humorística oral en Costa Rica», Revista Reflexiones 1, 86 (2007): 45-57 (47); Alberto Barrantes, «Caso Natividad Canda: Pendientes de Costa Rica para sanar resabios de xenofobia», Derecho al dia, 13 febrero, 2020, <https://derechoaldia.com/index.php/constitucional/constitucionaldoctrina/845-caso-natividad-canda-pendientes-de-costa-rica-para-sanar-resabios-de-xenofobia> .

6 Sherry E. Gapper, Manual de gestión terminológica (Heredia: Editorial Universidad Nacional, 2008) 13-88. 
Hasta abril de 2020 no se había registrado una traducción al inglés del cuento seleccionado, hecho que constituye en sí mismo una brecha para el acceso de la literatura centroamericana al público lector angloparlante que no domina el español. En el ámbito teórico, el trabajo contribuye al desarrollo del campo de la terminología en Costa Rica al ampliar, de forma modesta al menos, el volumen de publicaciones de esta índole temática.

\section{Reseña teórica}

\section{La terminología: ¿qué es y por qué se escogió?}

Aunque los conceptos alrededor de la terminología son muy variados y a menudo generales, nos acogemos a lo indicado por Juan C. Sager, quien la delimita como un campo y una práctica interdisciplina-ria «relacionada con la recopilación, la descripción y la presentación de términos, es decir, los elementos léxicos que pertenecen a áreas especializadas de uso en una o más lenguas» ${ }^{7}$. En la actualidad, la terminología asocia a una serie de disciplinas auxiliares, tales como la semántica, la lingüística aplicada, las ciencias de la información, la lexicografía y muchas más, por lo que no sorprenderá que en oca-siones la línea divisoria entre esta y otras áreas de especialización no se encuentre tan claramente definida. Según propone Sager, la terminología es comparable a la lexicografía, que busca «la doble finalidad de la recopilación general de información sobre el léxico de la lengua con la de suministrar información y, en ocasiones, in-cluso con un servicio de asesoramiento a los usuarios de la lengua»». Lo que hace a la primera distinta de la segunda es el propósito de la información recopilada, la formación de los encargados de esta tarea y, en gran medida, las metodologías de gestión terminológica utilizadas, explica el autor.

\footnotetext{
7 Juan C. Sager, Curso práctico sobre el procedimiento de la terminología (Madrid: Pirámide, 1993) 21.

8 Sager, 21.
} 
Para María Teresa Cabré, la terminología desempeña un papel medular en la traducción en general, aunque su importancia se torna crucial cuando nos enfrentamos con traducciones especializadas. El grado de necesidad y compromiso con la terminología varía según el nivel de especialización presente en los textos ${ }^{9}$. Aunque no siempre se reconoce, esto no suele ser distinto en muchos textos literarios, donde el autor recurre a diversas modalidades textuales para llevar a cabo un determinado propósito comunicativo ${ }^{10}$. Así, en «Abbott y Costello», el traductor afronta una crónica periodística que emplea diversas modalidades textuales (periodística, legal, médica, etc.), las cuales, a su vez, exhiben características terminológicas específicas donde se condensa gran contenido semántico.

Según Cabré, uno de los problemas con la traducción de este tipo de textos es que no siempre se cuenta con «terminología de referencia y menos aun de terminología codificada en glosarios o bancos de datos suficientemente actualizada para cubrir las necesidades del traductor $\rangle^{11}$. Por ello, debido al grado de especialidad presente a lo largo del relato que nos ocupa, el empleo de un enfoque terminológico resultó vital para una traducción acorde con el tono y el grado de especialización empleados en distintos apartados del TO. Si bien sabemos que existen diversos grados de especialización terminológica, incluso dentro de una misma modalidad textual, este análisis no busca profundizar en tal aspecto ${ }^{12}$. El análisis traductológico comprende términos técnicos, pero también comprende algunos que resultan problemáticos dada su polisemia en distintos ámbitos culturales.

9 María Teresa Cabré, «El traductor y la terminología: necesidad y compromiso»,Panace@: Revista de Medicina, Lenguaje y Traducción 1, 2 (2000): 2-4.

10 Alejandro Ulloa y Giovanna Carvajal, «Teoría del texto y tipología discursiva», Signo y pensamiento 53 (2008): 295-313.

11 Cabré, 3.

12 Una discusión análoga sobre el tema se encuentra en: Fredricka Stoller, «EAP Materials and Tasks», The Routledge Handbook of English for Academic Purposes (Nueva York: Routledge, 2016) 579. 


\section{Proceso de almacenamiento de datos terminológicos}

Según Gapper, gracias a los múltiples medios digitales y electrónicos disponibles para el almacenamiento de datos terminológicos, en la actualidad, el sistema de fichas bibliográficas supone una alternativa práctica y económica para el respaldo de los términos investigados ${ }^{13}$. Dependiendo del propósito de la gestión terminológica, existen tres tipos de fichas: la bibliográfica, la terminológica y la lexicográfica, y se elaboran de la siguiente manera (ver ejemplos completos en el apartado de la metodología):

Las fichas bibliográficas contienen la cita bibliográfica completa de cada una las fuentes consultadas; se utilizan en conjunción las terminológicas, en las que se recogen principalmente ejemplos del uso de cada término. Por su parte, las lexicográficas forman la base para cada una de las entradas de un glosario ${ }^{14}$.

En el proceso de confección de las fichas descritas anteriormente, se incluyen ejemplos de uso en contexto de fuentes tanto primarias (por ejemplo, textos paralelos provenientes de libros, folletos o artí-culos con uso real de los términos; fuentes orales como entrevistas, conversaciones con especialistas del campo que estudiamos; entre otras) como secundarias (diccionarios, glosarios, etc. $)^{15}$. De este modo, la traducción de las unidades léxicas seleccionadas contará con respaldo lingüístico, además de amplio, fundamentado en el contexto de especialización de la lengua de llegada.

\section{Análisis textual y extratextual}

«Abbott y Costello» forma parte de una colección de relatos publicados en el 2013, tanto en el libro Flores oscuras ${ }^{16}$ como en la

13 Gapper, 65.

14 Gapper, 66.

15 Gapper, 46-48.

16 Sergio Ramírez, «Abbott y Costello», Flores oscuras (México: Santillana, 2013) 188-203. 
revista Cultura de $\mathrm{pa}^{17}$. Se divide en siete partes: 1) Los hechos, 2) El occiso, 3) El shock hipovolémico, 4) Los perros, 5) Reconstrucción de los hechos, 6) La sentencia judicial y 7) Punto final. Busca informar, mediante esta modalidad discursiva, sobre los acontecimientos que culminaron con la muerte de Canda Mairena por el ataque de dos perros rottweilers. Está escrito en un tono formal y recurre a un registro culto a lo largo de la narración; sin embargo, el tono se vuelve informal, íntimo y apoyado por un registro coloquial, cuando los interlocutores son los parientes de Canda Mairena.

En cuanto a los elementos textuales, el cuento se forja en el registro de la crónica periodística, lo que da como resultado una presentación de los hechos en tono objetivo y sobrio. Se logra esto mediante escogencias léxicas particulares (de orden científico, médico, legal, entre otros), como la presentación de los hechos narrativos de forma imparcial y ajustando la estructura sintáctica a los distintos tipos textuales que subyacen la historia. Si bien estos rasgos no comprenden un carácter taxativo en la crónica periodística en general, Ramírez se ha valido principalmente de estos para dotar su obra de la veracidad típica del género ${ }^{18}$.

Con respecto a los elementos extratextuales, los factores culturales e históricos en que se inscribe la obra, la narración gira en torno a la muerte de Canda. Según indica el texto y los periódicos costarricenses The Tico Times ${ }^{19}$ y La Prensa ${ }^{20}$, así como los diarios nicaragüenses El Nuevo Diario ${ }^{21}$ y La Gente ${ }^{22}$, Canda habría entrado a robar a un taller automotriz y los perros lo atacaron y lo mordieron en repetidas ocasiones. El ataque se desarrolló durante

17 Sergio Ramírez, «Abbott y Costello», Cultura de paz 59 (2013): 28-34.

18 Nancy Salas Andrade, «La vigencia de un género: la crónica periodística», Revista de Comunicación 1 (2003): 78.

19 Leland Baxter-Neal, «Officials Criticized for Dog Attack Response», The Tico Times, 5 marzo, 2019, <https://ticotimes.net/2006/03/17/officials-criticized-for-dog-attack-response>.

20 Josué Bravo, «Suspenden por un año más último juicio por muerte de Natividad Canda», La Prensa, 10 abril, 2019, <https://www.laprensa.com.ni/2016/08/23/nacionales/2088191-juicio-natividad-canda>

21 Carlos Larios, «Muerte de Canda impune», El Nuevo Diario, 6 febrero, 2020, <https://www.elnuevodiario.com.ni/nacionales/266866-muerte-canda-impune/>.

22 Sinautor, «SepultanalnicadestrozadoporlosperrosenCostaRica»,LaGente, 21 febrero, 2020, <http://www.radiolaprimerisima.com/noticias/generica/1180/sepultan-al-nica-destrozado-por-los-perros-en-costa-rica/>. 
dos horas, y a pesar de que en el lugar de los hechos había dos guardias de seguridad, el dueño del taller mecánico y ocho policías, estos no procuraron rescatar a la víctima del ataque. Canda murió de un choque hipovolémico (i. e., pérdida grave de sangre y fluidos corporales) al llegar al hospital Max Peralta en Cartago, producto de la pérdida de sangre por las 197 heridas provocadas por los caninos ${ }^{23}$. Años después, en el juicio por la muerte de la víctima, los jueces dictaminaron, por mayoría de votos, que los perros no pudieron haber sido detenidos mediante disparos porque se movían más rápido que las balas ${ }^{24}$.

\section{Metodología de traducción}

El presente apartado esboza los fundamentos teóricos que rigen este estudio: la hermenéutica, disciplina encargada de interpretar ${ }^{25}$. De ella se deriva la investigación conceptual o teórica que, según Williams y Chesterman se define como la investigación que busca (1) definir y clarificar conceptos, (2) interpretar ideas, (3) relacionar conceptos y (4) introducir conceptos, metáforas, y marcos que permitan comprender de mejor manera el objeto de estudio $^{26}$. Este tipo de investigación se escogió debido a que, al realizar la traducción al inglés, en el TO se identificó una serie de términos, principalmente especializados, los cuales presentaron distintos grados de dificultad al trasladarlos a la lengua terminal. Dicho esto, detallamos a continuación la metodología utilizada para llevar a cabo la traducción de dichas unidades léxicas ${ }^{27}$ :

23 Ramírez, 198.

24 Ramírez, 202.

25 Jenny Williams y Andrew Chesterman, The Map: A Beginner's Guide to Doing Research in Translation Studies (Manchester: Routledge, 2002) 58. DOI: https://doi.org/10.4324/9781315760513.

26 Williams y Chesterman, 58.

27 Gapper, 32-77. 
1. selección de términos problemáticos en el TO y clasificación según especificidad temática, registro y contexto;

2. selección y recopilación de fuentes primarias y secundarias, así como el estudio de textos paralelos, para escoger las equivalencias léxicas en la lengua meta y mantener el estilo periodístico presente en el TO y

3. elaboración de fichas bibliográficas, terminológicas y lexicográficas para «almacenar la información obtenida sobre los términos investigados ${ }^{28} \gg$.

Para ejemplificar el resultado de esta metodología, véanse los tres tipos de fichas elaboradas durante el proceso de traducción del cuento. El cuadro 1 (basado en la ficha $n^{\circ} 21$ ) constituye el primer ejemplo de lo anterior.

Cuadro 1. Ejemplo de ficha lexicográfica

\begin{tabular}{|l|l|}
\hline Glosario «Abbott y Costello», \\
\hline Proyecto: «Abbott y Costello» & Género: científico \\
\hline Elaborado por: & $\begin{array}{l}\text { Fecha de elaboración: } \\
\text { 4 mayo, 2019 }\end{array}$ \\
\hline Término: manto freático & English equivalent: water table \\
\hline $\begin{array}{l}\text { Categoría o función } \\
\text { gramatical: frase nominal }\end{array}$ & Grammatical or functional category: noun phrase \\
\hline $\begin{array}{l}\text { Ejemplo (TO): } \\
\text { «...los sedimentos de los } \\
\left.\text { freático...» (1: } 194^{29}\right)\end{array}$ & $\begin{array}{l}\text { Examples: } \\
\text { "As ground water works its way through the soil, it } \\
\text { can pick up nitrogen and phosphorus and transport } \\
\text { them to the water table." (42: s. p.) } \\
\text { "Contaminants may reach ground water from } \\
\text { activities on the land surface, such as releases or } \\
\text { spills from stored industrial wastes; from sources } \\
\text { below the land surface but above the water table, } \\
\text { such as septic systems ..." (43: s. p.) }\end{array}$ \\
\hline
\end{tabular}

28 Gapper, 65.

29 Ramírez, 194. 
En cuadro 2 se ilustra el empleo de una ficha terminológica.

Cuadro 2. Ejemplo de ficha terminológica

\begin{tabular}{|l|l|}
\hline \multicolumn{1}{|c|}{ Manto freático } & \multicolumn{1}{c|}{ Water table } \\
\hline «...los sedimentos & "As ground water works its way through the soil, it \\
de los insecticidas & can pick up nitrogen and phosphorus and transport \\
penetraron el manto & them to the water table." (42: s. p.) \\
freático...» (1: 194) & $\begin{array}{l}\text { "Contaminants may reach ground water from } \\
\text { activities on the land surface, such as releases or spills } \\
\text { from stored industrial wastes; from sources below the } \\
\text { land surface but above the water table, such as septic } \\
\text { systems ..." (43: s. p.) }\end{array}$ \\
\hline
\end{tabular}

El cuadro 3 muestra una fichabibliográfica basada en lafichan ${ }^{\circ} 42$.

Cuadro 3. Ejemplo de ficha bibliográfica

\begin{tabular}{|l|}
\hline $\mathbf{N}^{\mathbf{0}} \mathbf{4 2}$ \\
\hline Equipo de redacción de United States Environmental Protection Agency. \\
(s. f.). «Where this occurs: Ground Water and Drinking Water». En \\
Environmental Protection Agency (EPA). Recuperado de: https://www.epa. \\
gov/nutrientpollution/where-occurs-ground-water-and-drinking-water. \\
Artículo de página web. Ver primer párrafo del artículo. \\
En línea \\
\hline 29 abril, 2019 / HSM \\
\hline
\end{tabular}

Se omitieron los hipervínculos agregados a las fichas originales, tanto en el término como en el número de ficha bibliográfica. Para mejor claridad, enumeramos los cincuenta términos seleccionados con el número de página y subtítulo donde pueden ser encontrados en el TO (ver Flores oscuras ${ }^{30}$ ), y además los resaltamos en negrita y bastardilla en el TT (anexo 1). Obtenidos los permisos de difusión académica por parte del escritor Ramírez Mercado, procedimos con

30 Ramírez, 191-203. 
la traducción del texto. La versión traducida puede consultarse en su totalidad en el anexo 2 .

\section{Análisis de la traducción}

Se ofrece un análisis terminológico del TT en torno a las unidades léxicas que más problemas representaron. Por asuntos de espacio, el análisis tendrá carácter sumario y se centrará en cinco de las 50 unidades escogidas, únicamente. Para cada caso, se incluye una referencia al grado de especialización terminológica, algunas fuentes utilizadas, las dificultades encontradas y un cuadro comparativo que contiene (1) los términos del TO, (2) ejemplos de uso en textos paralelos y (3) nuestra propuesta de TT.

\section{Ejemplo 1: manto freático}

En hidrografía, el término especializado manto freático (conocido también como capa freática) se emplea para describir una sección del subsuelo, por lo general en un acuífero, por donde discurre el agua. Para la traducción de este término, se utilizaron dos textos paralelos en la lengua terminal, entre ellos un artículo gubernamental de la Environmental Protection Agency de los Estados Unidos de América, donde dicha unidad léxica se emplea en contexto. La dificultad principal radicó en encontrar textos paralelos de fuentes confiables, que muestran un nivel de especialización equivalente al del TO. En el cuadro 4 se presenta una comparación de uso de este término en el TO y en el TT. 
Cuadro 4. Manto freático

\begin{tabular}{|c|c|c|}
\hline Término: manto freático & Equivalente en inglés: wate & r table \\
\hline $\begin{array}{l}\text { Ejemplo en contexto } \\
\text { (TO): Antiguamente } \\
\text { se sembraba también } \\
\text { algodón, cultivo que } \\
\text { envenenó sin remedio } \\
\text { las fuentes de agua, } \\
\text { pues los sedimentos } \\
\text { de los insecticidas } \\
\text { penetraron el manto } \\
\text { freático, de modo que } \\
\text { hasta la leche materna } \\
\text { se halla contaminada } \\
\text { de toxaclorofeno». } \\
\text { (Ramírez 194) }\end{array}$ & $\begin{array}{l}\text { Ejemplo en texto paralelo: } \\
\text { "Contaminants may reach } \\
\text { ground water from activities } \\
\text { on the land surface, such } \\
\text { as releases or spills from } \\
\text { stored industrial wastes; } \\
\text { from sources below the land } \\
\text { surface but above the water } \\
\text { table, such as septic systems } \\
\text {..." (43: s. p. })^{31}\end{array}$ & $\begin{array}{l}\text { Propuesta de TT: } \\
\text { "Due to former cotton } \\
\text { plantation activity, } \\
\text { water sources became } \\
\text { poisoned because } \\
\text { the sediments from } \\
\text { insecticides permeated } \\
\text { the water table, so } \\
\text { much that even breast } \\
\text { milk is contaminated } \\
\text { with toxaphene." (Ver } \\
\text { Anexo 2: } \text { el texto } \\
\text { traducido.) }\end{array}$ \\
\hline
\end{tabular}

\section{Ejemplo n. ${ }^{o}$ 2: licenciada}

Si bien no se trata de un término especializado, la dificultad central de la traducción de este término estriba en la naturaleza polisémica que adopta según su uso en distintas regiones. En Costa Rica y otros países de Hispanoamérica (México y Nicaragua), la voz licenciado puede referirse tanto a un profesional en derecho como a un grado académico posterior al bachillerato universitario, anterior al de maestría. De manera similar, el Diccionario de la lengua española registra seis acepciones de la palabra, entre las cuales se encuentra la de «Dicho de una persona: Que se precisa entendida», como también: «Hombre que vestía hábitos largos o traje de estudiante» ${ }^{32}$. En el ámbito especializado de la medicina veterinaria, el concepto se relaciona más bien con aquella persona que, por sus estudios o experiencia, posee las credenciales técnicas para realizar chequeos, llevar a cabo

31 Sin autor, «Where this occurs: Ground Water and Drinking Water», United States Environmental Protection Agency, 4 mayo, 2019, <https://www.epa.gov/nutrientpollution/ where-occurs-ground-water-and-drinking-water>.

32 Real Academia Española, Diccionario de la lengua española, 4 mayo, 2019, <https://dle.rae.es/ licenciado? $\mathrm{m}=$ form $>$. 
diagnósticos y tratar las enfermedades de los animales. Dentro de este marco conceptual, se recurrió a dos fuentes primarias que registraban el uso del término, entre ellas una página web que describe las labores de un licenciado en veterinaria (animal doctor) y los requisitos que se deben tener para estudiar y ejercer esta profesión ${ }^{33}$. El cuadro 5 compara el uso de este término en el TO y en el TT.

Cuadro 5. Licenciada

\begin{tabular}{|c|c|c|}
\hline Término: licenciada & Equivalente en ingle & és: animal doctor \\
\hline $\begin{array}{l}\text { Ejemplo en contexto (TO): } \\
\text { «La licenciada Valentina del } \\
\text { Socorro Camacho, veterinaria } \\
\text { y experta en conducta animal, } \\
\text { explicó el motivo por el que } \\
\text { los rottweiler no obedecieron } \\
\text { las órdenes de detenerse, una } \\
\text { vez que tenían cercada a la } \\
\text { víctima». (Ramírez 193) }\end{array}$ & $\begin{array}{l}\text { Ejemplo en texto } \\
\text { paralelo: } \\
\text { «An animal doctor } \\
\text { is a veterinarian } \\
\text { who performs } \\
\text { health checks, } \\
\text { diagnoses, and } \\
\text { treats any type of } \\
\text { animal». }(26: \text { s. p. })^{34}\end{array}$ & $\begin{array}{l}\text { Propuesta de TT: } \\
\text { «Animal doctor Valentina } \\
\text { del Socorro Camacho, } \\
\text { veterinarian and animal } \\
\text { behavior expert, explained } \\
\text { the reason the rottweilers } \\
\text { did not obey the orders to } \\
\text { stop once they had sieged } \\
\text { the victim». (Ver } \text { Anexo 2: } \\
\text { el texto traducido.) }\end{array}$ \\
\hline
\end{tabular}

\section{Ejemplo n. ${ }^{\circ}$ 3: peritos}

La dificultad en la traducción de este término se produce por la polisemia en la palabraperitos, ya que en distintas áreas de especialización se utiliza como sinónimo de experto, especialista e incluso licenciado, entre otras acepciones. En ciertos contextos técnicos y culturales, se habla de peritos de un banco, cuyo trabajo consiste en efectuar avalúos para estudios crediticios o, en el ámbito legal, también para referirse a personas o entes expertos en materia cultural, científica, académica, etc., según lo determine el caso en cuestión ${ }^{35}$. Sin embargo, en «Abbott y Costello», Ramírezutiliza peritos para referirse más bien a expertos en materia canina. Para resolver el problema de traducción, se consultaron

33 Sin autor, «How to Become an Animal Doctor in 5 Steps», Learn.org, 7 mayo, 2019, <https://learn. org/articles/Animal_Doctor_Become_an_Animal_Doctor_in_5_Steps.html $>$.

34 Sin autor, «Animal Doctor: Requirements for Becoming a Veterinarian», 7 mayo, 2019, <https:// study.com/articles/Animal_Doctor_Requirements_for_Becoming_a_Veterinarian.html $>$.

35 Diccionario del español jurídico, 25 mayo, 2019, ${ }^{<}$https://dej.rae.es/lema/perito-ta $>$. 
dos fuentes primarias, entre las cuales figuran un artículo reciente (2019) sobre razas de perros ${ }^{36}$. En el cuadro 6 se incluye la oración en contexto, tanto del TO como del TT.

\section{Cuadro 6. Peritos}

\begin{tabular}{|l|l|l|}
\hline Término: peritos & Equivalente en inglés: experts & \\
\hline Ejemplo en contexto & Ejemplo en texto paralelo: & Propuesta de \\
(TO): & «But we asked experts familiar & TT: «Each dog \\
«Cada uno tiene un & with nine expensive dog breeds & is estimated to \\
costo aproximado & to share with us an average price & cost around $\$ 500$, \\
de quinientos & range, why the price tags for those & according to experts \\
dólares, según & breeds may be so high and some & consulted». (Ver \\
peritos consultados». & of the beloved traits that each of & Anexo $2:$ el texto \\
(Ramírez 193) & them possess[es]». (117: s. p.) ${ }^{37}$ & traducido.) \\
\hline
\end{tabular}

\section{Ejemplo n. ${ }^{\circ} 4$ : toxa(cloro)feno}

El toxafeno es un insecticida clorado con alta cantidad de productos químicos. Se utilizó para control de plagas en las plantaciones de algodón; por su alto riesgo de toxicidad hoy se encuentra prohibido en muchos países, entre ellos los Estados Unidos y Nicaragua. A primera vista, traducir «toxafeno» pareciera ser un procedimiento de transferencia léxica hacia su equivalente en inglés «toxaphene». El problema se presentó debido a que el TO recoge un término que, en el mundo especializado de la química, no se encuentra registrado. Tal es el caso de toxaclorofeno, palabra empleada por el autor en la sección «El occiso», donde se describe la procedencia de Natividad Canda: «Natividad Canda nació el 13 de agosto de 1980 en Chichigalpa, en el occidente de Nicaragua [...]. Son las tierras más fértiles del país [...]. Antiguamente se sembraba también algodón, cultivo que envenenó sin remedio las fuentes de agua, pues los sedimentos de los insecticidas penetraron el manto freático, de modo que hasta la leche materna se halla contaminada de toxaclorofeno $»^{38}$.

36 Amy Jamieson, «Expensive dog breeds: What to know about them and their price tags», 25 mayo, $2019,<\mathrm{https}: / /$ www.care.com/c/stories/6111/the-top-10-most-expensive-dog-breeds-in-the-w/>.

37 Jamieson, párrafo 2.

38 Ramírez, 194. 
Después de una exhaustiva búsqueda en distintas revistas especializadas, así como de consultas con expertos en el campo de la química, se concertó una entrevista personal con el autor, quien pidió reemplazar el término original por toxafeno, nomenclatura especializada correcta para denotar lo que inicialmente había sido llamado toxaclorofeno en «Abbott y Costello»,. Realizadas estas aclaraciones, se procedió a buscar ejemplos de uso «toxaphene» en contexto, lo cual se puede apreciar en el cuadro 7 , donde se muestra la oración en el TO y un ejemplo del término utilizado por una fuente primaria.

Cuadro 7. Toxafeno

\begin{tabular}{|l|l|l|}
\hline Término: toxa(cloro)feno & \multicolumn{2}{|l|}{ Equivalente en inglés: toxaphene } \\
\hline $\begin{array}{l}\text { Ejemplo en contexto (TO): } \\
\text { «Antiguamente se sembraba } \\
\text { también algodón, cultivo } \\
\text { que envenenó sin remedio } \\
\text { las fuentes de agua, pues los } \\
\text { sedimentos de los insecticidas } \\
\text { penetraron el manto freático, } \\
\text { paralelo: } \\
\text { de modo que hasta la leche } \\
\text { materna se halla contaminada de } \\
\text { toxaclorofeno». }\end{array}$ & $\begin{array}{l}\text { (Ramírez 194) } \\
\text { one of the most } \\
\text { heavily used } \\
\text { pesticides... It was } \\
\text { used primarily to } \\
\text { control insect pests } \\
\text { on cotton and } \\
\text { other crops...» }\end{array}$ & $\begin{array}{l}\text { «De to former cotton } \\
\text { plantation activity, water } \\
\text { sources became poisoned } \\
\text { because the sediments } \\
\text { from insecticides } \\
\text { permeated the water table, } \\
\text { so much that even breast } \\
\text { milk is contaminated with } \\
\text { toxaphene». (Ver } \text { Anexo 2: } \\
\text { el texto traducido. })\end{array}$ \\
\hline
\end{tabular}

\section{Ejemplo n. ${ }^{\circ}$ 5: omisión de auxilio}

La omisión de auxilio (a menudo referida como omisión de socorro u omisión del deber de socorro) es un delito imputado a una persona por reusar, de manera voluntaria, prestar ayuda inmediata a otra persona cuya seguridad corporal se encuentra en riesgo. En el contexto jurisprudencial de Costa Rica, la omisión de auxilio se puede entender de la siguiente manera:

conducta ilícita que comete quien encuentra perdida o desamparada a una persona menor de diez años, $\mathrm{o}$ a una persona herida o amenazada

39 Sin autor, «Compound Summary», U.S. National Library of Medicine, 22 mayo, 2019, <https:// pubchem.ncbi.nlm.nih.gov/compound/5284469\#section=Information-Sources>. 
de un peligro cualquiera y omite prestarle el auxilio necesario según las circunstancias, cuando puede hacerlo sin riesgo personal ${ }^{40}$.

En el proceso de traducción de «Abbott y Costello», se encontraron diversas fuentes que utilizaban distintos términos aproximativos para denotar el crimen en cuestión. Entre los utilizados en algunas noticias se encuentran failure to act e improper omission; sin embargo, las dos traducciones más utilizadas en los contextos consultados son omission of aid y crime of omission. Se eligió omission of aid debido a que la oración traducida ya contenía la palabra crime: «Lawyer Fernando Sotela [...] filed a lawsuit [...] for the crime of simple homicide in concurrence for omission of aid». De este modo, la justificación detrás de la escogencia del término se fundamenta en la estilística, más que en la lexicografía misma. El cuadro 8 ofrece un ejemplo en contexto del término traducido al inglés.

\section{Cuadro 8. Omisión de auxilio}

\begin{tabular}{|c|c|c|}
\hline & \multicolumn{2}{|l|}{ Equivalente en inglés: omission of aid } \\
\hline $\begin{array}{l}\text { Ejemplo en contexto } \\
\text { (TO): } \\
\text { «El licenciado } \\
\text { Fernando Sotela }[\ldots] \\
\text { interpuso }[\ldots] \text { una } \\
\text { querella }[. . .] \text { mediante } \\
\text { la cual acusó por el } \\
\text { delito de homicidio } \\
\text { simple en concurso de } \\
\text { omisión de auxilio al } \\
\text { dueño del taller }[. . .] » \\
\text { (Ramírez 201) }\end{array}$ & $\begin{array}{l}\text { Ejemplo en texto paralelo: } \\
\text { «According to the Criminal Code: } \\
\text { Article 144, Omission of Aid is } \\
\text { 'Finding a child under ten years of } \\
\text { age, or an injured person or a person } \\
\text { threatened with any danger, lost } \\
\text { or helpless, and failing to provide } \\
\text { necessary assistance according to } \\
\text { the circumstances, as long as it can } \\
\text { be done without personal risk'». }\end{array}$ & $\begin{array}{l}\text { Propuesta de TT: } \\
\text { «Lawyer Fernando Sotela } \\
{[\ldots] \text { filed a lawsuit }[\ldots]} \\
\text { whereby he accused the } \\
\text { owner of the shop }[\ldots] \\
\text { for the crime of simple } \\
\text { homicide in concurrence } \\
\text { for omission of aid». } \\
\text { (Ver Anexo } 2: \text { el texto } \\
\text { traducido.) }\end{array}$ \\
\hline
\end{tabular}

40 Diccionario usual del Poder Judicial, 25 mayo, 2019, <https://digesto.poder-judicial.go.cr/index. $\mathrm{php} / \mathrm{dicc} /$ 44259:omisi\%C3\%B3n-de-auxilio>.

41 Equipo de redacción de A.M. Costa Rica, «Prosecutor files charges against three linked to death of four U.S. citizens during rafting», 20 febrero de 2020, <http://amcostarica.com/Prosecutor $\% 20$ files $\% 20$ charges $\% 20$ against $\% 20$ three $\%$ 20linked $\% 20$ to $\% 20$ death $\% 20$ of $\% 20$ four $\% 20$ U.S. $\% 20$ citizens\%20during\%20rafting.html>. 


\section{Conclusiones y reflexiones}

En primer lugar, la traducción de una crónica periodística como la aquí abordada requiere de una indagación extratextual previa, de modo que se pueda ubicar el TO dentro de un sistema histórico, político y cultural definido, como indica el teórico Jeremy Munday ${ }^{42}$. El traductor podrá abocarse a la traducción de los rasgos lexicográficos y estilísticos del TO, ocuparse de aspectos fraseológicos y ortotipográficos e idear los mecanismos para verter todo lo anterior en el sistema lingüístico de la lengua de llegada. En segundo lugar, si bien el estudio de textos paralelos no es una práctica exclusiva en la traducción de textos literarios, ello se torna una tarea aún más vital en la traducción del relato periodístico, ya que por su brevedad este tipo de textos pueden condensar diversas modalidades discursivas dentro de un mismo hilo narrativo. Así, en un relato como «Abbott y Costello», el traductor debe contar con conocimientos terminológicos del lenguajemédico, legal, incluso coloquial.El enfoque terminológico empleado facilitó la traducción de terminología tanto especializada como de uso genérico que presentaba variaciones regionales, con el respaldo de fuentes primarias que ejemplifican el uso de dichas unidades léxicas en la lengua meta.

Hasta abril de 2020 no se había registrado traducción al inglés del texto en cuestión. Por lo tanto, nuestro trabajo representa, a nivel práctico, un esfuerzo por potenciar la traducción de literatura centroamericana al idioma inglés, con el valor agregado de que al hacerlo se toca un tema de corte mundial: los suplicios de los inmigrantes que dejan su patria para buscar la fortuna que su tierra les niega, y que hallan a menudo tragedias más allá de toda sospecha ${ }^{43}$. En el plano de lo teórico, el artículo supone un avance para la terminología y la traductología en Costa Rica, ya que amplía el catálogo de publicaciones académicas que abordan estas áreas temáticas. Asimismo, siquiera modestamente, se

42 Jeremy Munday, Introducing Translation Studies: Theories and Applications (Londres: Routledge, 2013) 171. DOI: https://doi.org/10.4324/9780203121252.

43 Ver: José Ángel Vargas, «Migración y deconstrucción del imaginario costarricense en “Abbott y Coste1lo”, de Sergio Ramírez», Revista Letras, 2, 64 (2018): 13-26. DOI: https://doi.org/10.15359/rl.2-64.1. 
realiza un aporte colateral para los estudios literarios, específicamente en la difusión de la literatura de fronteras a un público anglófono.

Si se realiza una evaluación apresurada del relato, podría pensarse que estamos ante una crítica contra la sociedad costarricense por su indolencia ante la tragedia de su vecino (el nicaragüense); sin embargo, un criterio más razonado develará que se trata de una denuncia contra algo mucho más universal: la ley de la supervivencia del más fuerte. En este orden de aniquilación, el clásico tópico de la explotación del hombre por el hombre (abordado magistralmente en La compuerta número 12, del escritor colombiano Baldomero Lillo) es llevado a sus peores extremos para colocarnos frente a frente con la destrucción del más débil a manos del más fuerte. En este y muchos otros sentidos, «Abbott y Costello» es un grito de alerta frente a los juegos arrasadores del poder. Y aunque redundar sobre estas realidades no parezca ayudar a mejorar la condición del género humano, al menos nos queda aquella sentencia de John Donne (citado por Ernesto Sábato) que nos recuerda la misión de toda gran literatura: «despertar al hombre que viaja [dormido] hacia el patíbulo ${ }^{44}$.

En el plano traductológico, lo anterior es únicamente posible mediante el análisis terminológico de rigor efectuado, pues es esto precisamente lo que posibilita la aproximación léxica requerida para que la crónica periodística del texto original sea crónica periodística en la lengua terminal, para que las unidades léxicas cuenten con un equivalente respaldado por fuentes primarias contextualizadas $y$, finalmente, para que el lector meta reciba un mensaje tan parecido como sea posible a lo que el autor intentó transmitir en su texto original.

\section{Recomendaciones}

Cuando se trata de traducción de crónica periodística, el primer elemento vital es la consulta de fuentes primarias en la lengua terminal, pues es así como se desarrolla una consciencia del tono, el registro

44 Ernesto Sábato, El escritor y sus fantasmas (Madrid: Editorial Aguilar, 1963) 22. 
y la terminología presentes en este tipo de textos. En el caso que nos ocupa, uno de los primeros artículos base utilizados fue «Officials Criticized for Dog Attack Response», publicado por The Tico Times, y que refiere algunos avances en torno a investigaciones generadas por la muerte de Canda Mairena.

Es preciso emprender una búsqueda minuciosa en inglés de la terminología especializada del TO. Aunque esto parezca axiomático dentro de la labor traductológica, conviene recalcarlo, ya que un eventual desacierto en la traducción de dichos términos induciría a errores semánticos (incluso pragmáticos) que alejarían al TT del original. Al tratarse también de un tema polémico para los países involucradas, la precisión terminológica jugará un papel decisivo en la transmisión de un mensaje fiel al original, y a los hechos representados.

Finalmente, conviene desarrollar una sensibilidad hacia el ritmo de la prosa, tanto en el TO como en el TT. Esto obedece a un principio literario que Gabriel García Márquez llamó «la carpintería» ${ }^{45}$, concepto relacionado con la libertad de utilizar ciertas palabras como cuñas para mantener la cadencia de lo escrito y conseguir así la atención de quien lee. Esto hace que el texto se lea de corrido o pausadamente, según se desee; que el lector sienta su «latido», como decía Julio Cortázar ${ }^{46} ;$ y que por medio de esta arquitectura sintáctica se llegue al mensaje que el autor busca transmitir mediante los hechos narrativos representados. Para desarrollar esta destreza es preciso estar dispuestos a transgredir, aunque sea con moderación, cuando sea necesario, algunas reglas que la gramática normativa suele clasificar como intransgredibles. Si bien en materia terminológica nuestro estudio tendió a la equivalencia, en materia sintáctica y gramatical nos permitimos algunas licencias para dar con esa «carpintería» y ese «latido» sugeridos por los dos novelistas hispanoamericanos.

45 Álvaro Santana Acuña, «En la carpintería literaria», El País, 27 enero, 2020, <https://elpais.com/ cultura/2017/12/12/actualidad/1513111845_019920.html >.

46 Julio Cortázar, «Musicalidad y humor en la literatura», Clases de literatura: Berkeley, 1980 (Barcelona: Debolsillo, 2016) 153. 


\section{Anexos}

\section{Anexo 1. Términos seleccionados}

\section{Los hechos}

1. Madrugada (191)

2. Tiritaba (191)

3. Camionetas de tina (192)

4. Jirones del pantalón (192)

5. Peritos (193)

6. Licenciada (193)

7. En jauría (193)

8. Piel lustrosa (193)

9. Se afanan (193)

10. Enhiesta (193)

\section{El occiso}

11. Cordillera volcánica (194)

12. Manto freático (194)

13. Toxaclorofeno (194)

14. Deficiencia renal (194)

15. Cuadrillas (194)

16. Sosteniéndose la quijada (195)

17. Cause de lluvia (195)

18. Ataúd recubierto de peluche (196)

19. Asaltos en la vía pública (198)

\section{El shock hipovolémico}

20. Shock hipovolémico (198)

21. Volumen de la sangre (198)

22. Temblor (198)

23. Ritmo respiratorio (199)

24. Baja actividad celular (199) 
25. Muerte clínica (199)

26. Muerte cerebral (199)

\section{Los perros}

27. Cruz (199)

28. Constitución musculosa (199)

29. Radio (199)

30. Cierre en tijera (199)

31. Incisivos superiores (199)

32. Cubrir sin fisuras (199)

33. Incisivos inferiores (199)

34. Trufa (199)

35. Papada (199)

\section{Reconstrucción de los hechos}

36. Reconstrucción de los hechos (200)

37. Predio (200)

38. Expertos en balística y planimetría (200)

39. Representación (200)

40. Fiscal (200)

41. En perjuicio de la propia víctima (201)

\section{La sentencia judicial}

42. Querella (201)

43. Ministerio Público (201)

44. Delito de homicidio simple en concurso de omisión de auxilio (201)

45. Acusación penal (202)

46. Sentencia firme (202)

47. Voto contrario (202)

\section{Punto final}

48. Acta forense (203)

49. Occiso (203)

50. Cinturón de vaqueta (203) 


\title{
Anexo 2. Texto traducido
}

\section{Abbott and Costello ${ }^{47}$}

\author{
By Sergio Ramírez
}

\section{The Facts}

Natividad Canda Mairena, twenty-five years old, died on November 10, 2005, mangled by two rottweilers that mauled him during the hours before dawn.

His arms, elbows, legs, ankles, abdomen and chest were torn by bites, and the wounds on the elbows and ankles were so deep that the bones were left exposed. According to witnesses, when, after about two hours at the mercy of the dogs Canda was finally released, he uttered the following words: "Throw something on me 'cause I'm cold," or "throw me a blanket 'cause I'm cold." He was shivering uncontrollably. He was still alive when he was admitted to Max Peralta Hospital in the city of Cartago, but he died minutes after entering the emergency room as a result of severe blood loss.

At around 12:20 a.m., Canda had furtively jumped the perimeter wall of the facilities of the auto repair shop La Providencia, near the Los Gemelos Bridge, in the company of Carlos Andrés Rivera, alias "Banano," aiming to steal something, according to the police report. Both dogs focused on Canda only, as Rivera managed to escape by jumping back over the wall. Rivera was later arrested and taken to the Cartago jail.

It was the on-duty security guard Juan Francisco Picado who released the dogs when he noticed the presence of strangers. "They normally set the dogs loose after nine, but that night they were expecting a truck to be repaired the next day. That's why the dogs were locked in their cage," he said.

47 Sergio Ramírez, «Abbott y Costello», Flores oscuras (México: Santillana, 2013) 188-203. Traducción de Lindsay Chaves Fernández y Henry Sevilla Morales. 
The owner of the shop, Alejo Sanabria, showed up almost immediately after being telephoned, at around 12:40 a.m. About twenty minutes later, eight policemen arrived on a pickup truck. Sergeant Feliciano Ortuño, chief of the police patrol, said they studied the situation and decided not to shoot the dogs because they feared wounding Canda; Manuel Goyzueta, another security guard, agreed, showing the journalists shreds of the victim's pants. "I fired six shots in the air to scare them away, but they became more enraged," he said. One of the dogs is called Abbott, the other Costello. According to Goyzueta, it was Abbott who seized Canda and dragged him 25 meters away.

The rescue attempt by the Red Cross and the Firefighter Corps did not begin until 1:40 a.m. According to rescue worker Andrés Quirós, a total of 3,786 liters of pressurized water were used, and it was thanks to the powerful hoses that the dogs finally retreated.

The two rottweilers returned to their cages by themselves after Canda was taken to the hospital; then they were locked up again. Each dog is estimated to cost around $\$ 500$, according to experts consulted. Costa Rica's Health Ministry decided the dogs would not be euthanized after ruling out any chance of rabies.

Animal doctor Valentina del Socorro Camacho, veterinarian and animal behavior expert, explained the reason the rottweilers did not obey the orders to stop once they had sieged the victim. "The dogs were out of control; when they run in a pack, their animal instinct intensifies. When faced with the attack of two or more animals of this kind, a victim has no chance of surviving," the expert stated.

A video of the attack was recorded and is now available on YouTube: http://www.youtube.com/watch? $\mathrm{v}=$ YKrqZpD6VmI ${ }^{48}$. As shown in the video, both dogs are solid black=and have a glossy coat, and in the light of a strong flashlight that cuts through the darkness of the night, they are seen pouncing tirelessly on Canda's body, which is lying on the tall grass while a man in a brown jacket, who may well

48 Vídeo no disponible al 5 de marzo de 2020. 
be one of the guards of the shop, or its owner, remains with his back turned a few steps away. Then the body is dragged from one side to another by the dogs, and one of them sinks his fangs into the victim; the other watches with his head raised. It is not possible to tell which is Abbott and which is Costello. The man in the brown jacket remains at the scene, his back still turned.

\section{The Deceased}

Natividad Canda Mairena was born on August 13, 1980 in Chichigalpa, Chinandega, in the western region of Nicaragua, where temperatures reach up to $40{ }^{\circ} \mathrm{C}$ during the dry season. These are the most fertile lands of the country, located on a plain that stretches from the Maribios mountain range to the coast of the Pacific Ocean. The region is suitable for growing sugarcane, peanuts, soybeans, bananas and sesame. Due to former cotton plantation activity, water sources became poisoned because the sediments from insecticides permeated the water table, so much that even breast milk is contaminated with toxaphene. ${ }^{49}$

Canda's family currently lives in the "Modesto Palma" neighborhood, Chichigalpa. Natividad was the youngest of nine brothers and had remained single. His father died of a chronic kidney disease caused by the constant dehydration sugarcane cutters are exposed to, since they do their job under the bare sun and after the cane fields have been burned off, and that makes the temperature rise even more. Thus, the household responsibility was left to his wife Juana Francisca Mairena alone. To provide for her children, she worked cutting sugarcane, like any of the men in the harvesting crews, and as a maid, cooking, washing and ironing. In 1993, two of her sons (Antonio, 20, and Natividad, 13 back then) decided to try their fortune in Costa Rica, just as thousands of other illegal immigrants do.

49 Según comunicado personal de Sergio Ramírez, «toxaclorofeno» era el nombre del «insecticida clorado que producía la fábrica Penwalt en Nicaragua». Aunque en el TO se emplea «toxaclorofeno», el autor solicitó reemplazarlo por «toxafeno» en el TT, dado que «toxaclorofeno» no figura dentro de la terminología profesional de revistas y manuales especializados sobre el tema. 
According to his brother César Augusto, Natividad was deported several times, but he always managed to cross the border again through the blind spots which he knew like the palm of his hand. It had become something of a sport to him. "Why would I stay here and be one more mouth to feed?" he would tell his family every time he returned, only to go back. They would search for him at dawn, but he already would be gone. He had a strong-willed character. "The truth is that in Nicaragua, in addition to the lack of work opportunities, the wages are a joke. In Costa Rica, you get paid better salaries, so people leave the country with that hope," adds César Augusto, also a sugarcane cutter like his parents, and who, during the layoff time on the neighboring sugar plantation, spends all day, as he says, "twiddling his thumbs."

Juana Francisca Mairena was 68 years old when Natividad was killed. The roughness of life makes her look older: lean, hunched, dark-skinned, as if the sun had consumed and burned her, although her hair is barely streaked with gray. Four of her children, Margarito Esteban (38), César Augusto (36), Juana Francisca (35), and María Esperanza (32) live on the same neighborhood street. The street is not paved and looks more like a streambed where the weeds grow uncut. After many formalities, the family managed to get the remains of Natividad repatriated. Collecting the money as they could, the family sent the mother to bring the body back which arrived in a modest gray cloth-covered coffin.

On the first anniversary of his death, a Christian mass was celebrated in the parish of San Blas. To that date, both the mother and the sisters have still kept rigorous mourning. "He was never alone, but they left him to die: two full hours with those two beasts tearing him apart and nobody lifted a finger to get them off him, you see? What a sin!" says his sister María Esperanza while shaking her head with affliction, sitting on a plastic-woven rocking chair in his mother's house. It is a house patched together with wood planks, which you reach by climbing a cliff. Through the slits of the planks, smoke escapes from 
the kitchen stove. Down that cliff the cloth-covered coffin went, carried by the neighbors into the cemetery of Chichigalpa.

The family learned thenews through a phone call from Antonio, who works at a supermarket in San José. All he told them was that Natividad was dead, but he did not dare tell them that he had been torn apart by some dogs. They didn't find out until they saw the images on television in Nicaragua. "We could not believe that ragdoll the dogs were shaking to and fro at their pleasure was him," says María Esperanza.

"My brother was terrified of dogs," she adds. "He developed that fear from childhood, when a meek-looking German Shepherd bit him in the face and scarred him for life. The dog was tied up at the door of the house of one of the boiler technicians at the sugarcane mill, and my brother went up, all naïve, to pet him. Back then, Natividad was about eight years old, and he had just received his first Holy Com-munion at the church of San Blas. That day after the mass, my mom took him to the Chichigalpa park so he could have a picture taken by a street photographer, dressed in white and with a big candle (bigger than him) in his hand."

"The neighbors of the shop told my mother that while the dogs gnawed on and eventually killed my brother, some policemen who had just arrived watched the scene from afar, while others went back to the patrol car where they started to listen to the radio," says another of Canda's brothers, Margarito Esteban. Then he added, "When you publish this story, be sure to write that we are very grateful to Mr. Sotela, the lawyer who filed the prosecution without charging a single penny, moved by my mom's poverty."

Harold Fallas, a Costa Rican friend of Natividad, recounted that he [Canda] used to sleep under bridges, or wherever the night found him, and that to avoid being reported as undocumented, he pretended to sound Costa Rican when he spoke, and said that his family was from Tres Equis, Turrialba. In Los Diques, Cartago, where Canda once lived, they used to call him Nati. "He was the quiet type, not a troublemaker at all," said Bautista Lagos, a neighbor from that town. 
According tojudicial information gathered by the daily LaNación, in the year 2005 alone, when Natividad was killed, he had been subpoenaed eight times before the Courts of Justice, suspected of motor vehicle theft, burglary and stealing home appliances, street robberies, drug possession and consumption, and power and telephone line theft.

\section{The Hypovolemic Shock}

It was while lying on the bed of one of the two police trucks where he was to be taken to the hospital in Cartago that Natividad Canda said: "Throw something on me "cause I'm cold," or "throw a blanket on me "cause I'm cold." According to the official medical report, a total 197 bites were identified on his body. How much blood can escape through so many wounds caused by sharp fangs, over a period of two uninterrupted hours, is something that cannot be estimated, but, at any rate, it is more than enough to cause a hypovolemic shock.

A hypovolemic shock occurs when the body has lost a fifth or more of the normal volume of circulating blood. One of the main symptoms is the intense sensation of cold caused by severe hypothermia, which [in humans] consists of a decrease in body temperature below $31^{\circ} \mathrm{C}$. By then, the heart's pumping capacity is drastically weakened, and the functioning of other vital organs hindered. In addition to the intense cold, there is a state of anxiety that produces anguish, agitation and confusion of the senses, and uncontrollable shivering, followed by general weakness and loss of coordination, drowsiness, and decreased respiratory rate and pulse, until the person reaches lethargy, coma, and as a consequence of low cell activity, clinical death, and ultimately brain death.

\section{The Dogs}

The male rottweiler usually weighs between 110 and 120 pounds and measures between 61 and $68 \mathrm{~cm}$ at the withers. It has a large-sized head, solidly muscled body, muscular neck as well as moderate length, short snout, and iron jaws. The force of his bite is 300 pounds 
per square inch. The breed is armed with 42 scissor bite teeth, so that the upper incisors touch the inner surface of the lower ones entirely.

The nose is broad, well-shaped and always black, with relatively large nostrils. The eyes are medium-sized, almond-shaped and dark brown, and the ears are droopy, v-shaped, and widely separated. If it has saggy jowls or skin hanging from the throat, it is considered a bad specimen. The tail is usually cut when it is still a puppy, leaving only one or two vertebrae; the International Canine Association (ICA) standards prohibit full amputation.

Although rottweilers are used mainly for defense and protection, care and use manuals describe them as friendly, cheerful, meek, faithful, obedient, willing to learn and work on various tasks. In the canine classification ratings, the rottweiler is not present in the top ten for aggressiveness, but it is among the ten keenest, smartest creatures with a good sense of judgement. The ICA considers them ideal as family and property guardians.

\section{Reconstruction of the Facts}

On the night of Friday, November 25, 2005, judicial authorities carried out the reconstruction of the facts on the property of the auto repair shop La Providencia. About forty people participated in this reconstruction, including judges, prosecutors, forensic technicians, ballistics and planimetry experts, lawyers for each of the parties, members of the Judicial Investigation Agency, the police officers involved, as well as members of the Red Cross and members of the Fire Department who carried out the rescue. Two trained rottweiler dogs played Abbott and Costello, which stayed in their cage at all times while the procedure lasted, and a mannequin represented Canda, dressed in clothes similar to those he wore at the time of the attack. His partner Carlos Andrés Rivera, alias "Banano," played the role of himself in the crime-scene reconstruction, as did the owner of the shop, and the two security guards on duty. 
The purpose of requesting a competent court to reconstruct the events where they occurred - as proposed by the case's prosecutor Pablo de Jesús Peralta — was to determine if, while Canda was being attacked by the dogs, people present at one time or another could have done something to remove or stop the dogs; or if, on the contrary, the extreme aggressiveness they showed was a clear impediment to carrying out any action, including the firing of guns, and whether doing so they would have hit the victim instead.

The dogs, always at the orders and under the care of their trainer, attacked the mannequin until they mauled it to pieces, while the people involved were placed in the same spots they declared to have been on the day of the events investigated. The procedure lasted less than the real events.

\section{The Sentence}

Lawyer Fernando Sotela, on behalf of Juana Francisca Mairena widow-of-Canda, filed a lawsuit on November 15, 2005, before the Public Prosecutor of Cartago, whereby he accused the owner of the shop La Providencia, Alejo Sanabria, and the on-duty security guards Manuel Goyzueta and Juan Francisco Picado, for the crime of simple homicide in concurrence for omission of aid. He also accused the eight officers of the Public Force of committing the same crime, "for their manifest impassibility."

For his part, prosecutor Peralta only filed criminal charges for omission of aid against two of the members of the police force, Gamaliel Urbina and Yader Luna, "who, despite the urgency of the events, moved away towards one of the patrol cars and turned to talking, drinking coffee from a thermos, and supposedly listening to a music program on the radio."

The Cartago Court of Justice, composed of Judges Maribel Zeledón, Clarisa Chan and Rosaura Pacheco, by means of a final judgement handed down at ten o'clock on the morning of January 14 , 2006 , by a majority of two votes against one, acquitted from criminal 
liability, the owner of the shop, Alejo Sanabria, and the on-duty security guards, Manuel Goyzueta and Juan Francisco Picado, as well as the eight agents of the Public Force, dismissing in all its parts the charges filed against each one of them.

The duly justified dissenting opinion came from Judge Maribel Zeledón, who pointed out several inconsistencies and legal contradictions in the majority decision: "The expert opinion of a veterinarian was taken into account as the basis of the judgment, stating that a rottweiler jumps faster than the speed of a shot. So, according to this criterion, it is impossible for a bullet to reach the dog while on the move, an absurd assertion that seeks to justify the negligence of those whose duty was to shoot the enraged animals and did not, since a pistol bullet, like the ones used by the police, travels at 340 meters per second, a speed similar to that of sound, which in the Earth's atmosphere is 343.2 meters per second, and which no animal, no matter how fast, may ever reach."

\section{The End Point}

The forensic report records that in the morgue of the hospital in Cartago the following garments were removed from the deceased's body: "a pair of well-worn white sports shoes, a pair of green socks, a pair of extremely ragged jeans, a cowhide belt, and a light-blue cotton t-shirt with the Cáritas International logo, all of them with abundant bloodstains."

The same report states that in one of the pockets of his jeans a plastic wallet was found; it contained three bills of 50 colones $^{50}$, a prepaid telephone card, and folded in two, a rather tattered photo of a child holding a first communion candle.

Managua, January 2012

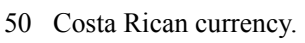

